\title{
Supplementary material to: 'A multi-level canopy radiative transfer scheme for ORCHIDEE (SVN r2566), based on a domain-averaged structure factor'
}

November 14, 2016

(McGrath M.J., Ryder J., Pinty B., Otto J., Naudts K., Valade A., Chen Y., Weedon J. and Luyssaert S.) 


\section{List of Figures}

S1 Two level fluxes of transmission (TRAN), total albedo (ALB), top of the canopy (CAN ALB) and absorption by the soil (SOIL ABS) as a function of the one level results for broad wide range of input parameters (see table 2). Different fluxes are represented by different colours. This figure corresponds to the REAL dataset (Table 2 in the main text defines the range of input values of each dataset). The equivalent plot for the ALL dataset is Figure 4 in the main text. . . . . . . . . S4

S2 Signed differences for various canopy fluxes of transmission (TRAN), total albedo (ALB), top of the canopy (CAN ALB) and absorption by the soil (SOIL ABS), between the two level results and the one level results $\left(\phi_{2}-\phi_{1}\right)$ as a function of the one level results for a broad range of input parameters. This figure corresponds to the REAL dataset (Table 2 in the main text defines the range of input values of each dataset). The equivalent plot for the ALL dataset is Figure 3 in the main text. S5

S3 Signed difference for various canopy fluxes of transmission (TRAN), total albedo (ALB), top of the canopy (CAN ALB) and absorption by the soil (SOIL ABS), between the two level results and the single level results $\left(\phi_{2}-\phi_{1}\right)$ as a function of the fraction of LAI in the top level for a broad range of input parameters. This figure corresponds to the REAL dataset (Table 2 in the main text defines the range of input values of each dataset). The equivalent plot for the ALL dataset is Figure 4 in the main text. . . . . . . . . . . . . . . S6

S4 Marginal values of each of the independent input variables against the fraction of points for which the absolute difference of $\left(\alpha_{\text {collim }}^{\text {mult }}-\alpha_{\text {collim }}^{\text {single }}\right)$, is less than 0.01 (red), 0.02 (green) and 0.03 (orange) for the 10 level multi-layer model, and below 0.01 (blue) for the 2 level multilevel model. . . . . . . . . . . . S7

S5 Marginal values for each of the independent input variables against $n_{\text {steps }}$, the total number of iterations required to arrive at a solution, for the ALL dataset, for 2 level model (blue) and the 10 level model $($ red). . . . . . . . . . . . . . . S8 
S6 Marginal values of each of the independent input variables against the fraction of points for which the absolute difference of $\left(\alpha_{\text {collim }}^{\text {multi }}-\alpha_{\text {collim }}^{\text {single }}\right)$, is less than 0.01 for the REAL value dataset (blue), and the ALL value dataset (red). . . . . . . . . . . . S9

S7 Marginal values for each of the independent input variables against $n_{\text {steps }}$, the total number of iterations required to arrive at a solution for the REAL value dataset (blue), and the ALL value dataset $($ red $) \ldots \ldots \ldots \ldots$. . . . . . . . . . S10 


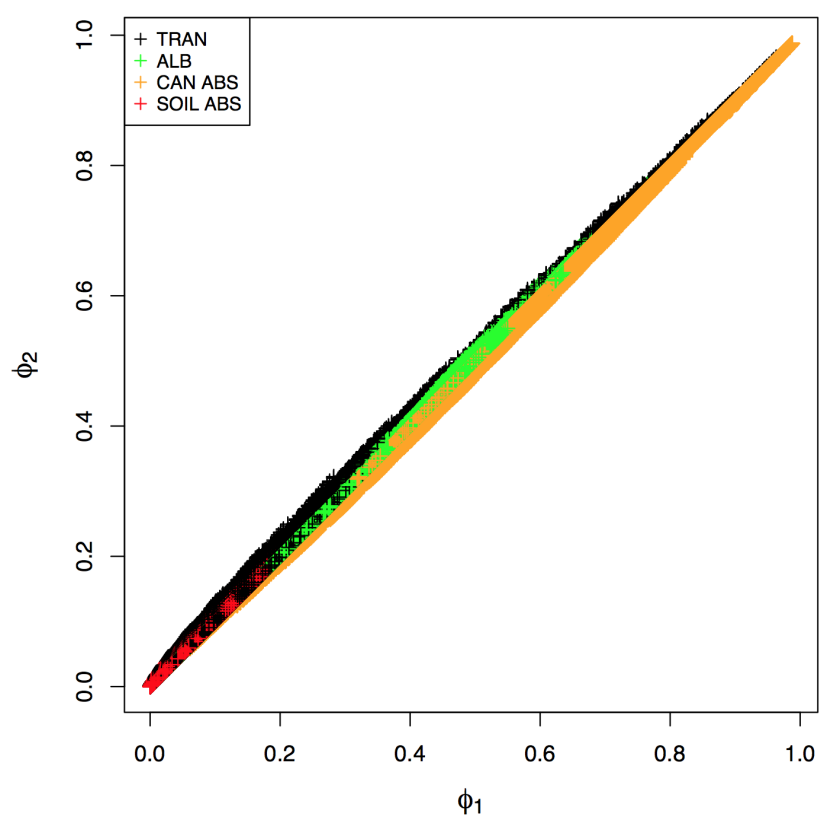

Figure S1: Two level fluxes of transmission (TRAN), total albedo (ALB), top of the canopy (CAN ALB) and absorption by the soil (SOIL ABS) as a function of the one level results for broad wide range of input parameters (see table 2). Different fluxes are represented by different colours. This figure corresponds to the REAL dataset (Table 2 in the main text defines the range of input values of each dataset). The equivalent plot for the ALL dataset is Figure 4 in the main text. 


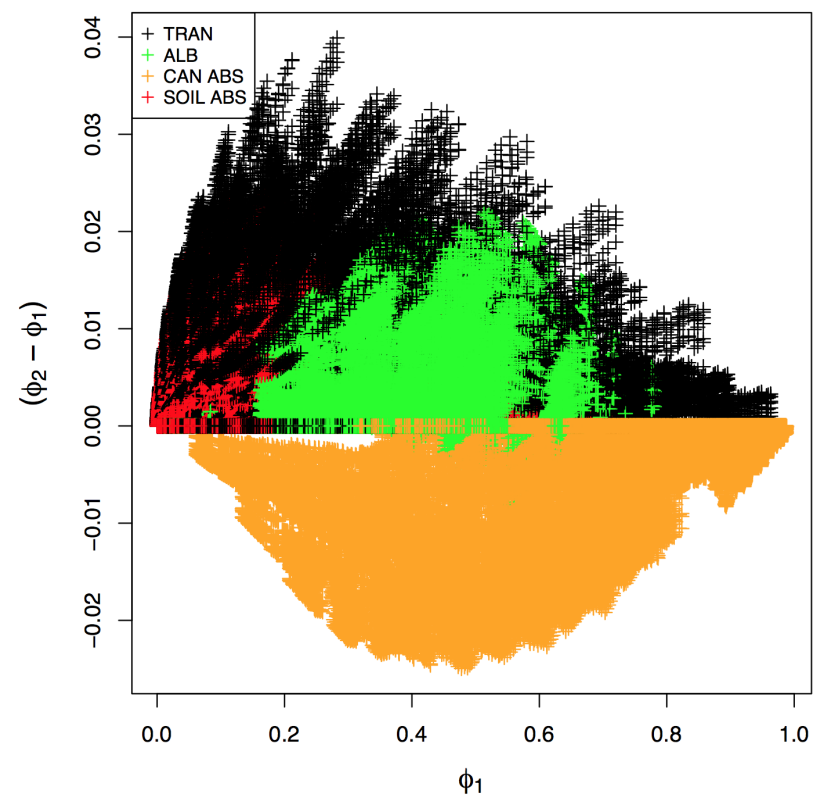

Figure S2: Signed differences for various canopy fluxes of transmission (TRAN), total albedo (ALB), top of the canopy (CAN ALB) and absorption by the soil (SOIL ABS), between the two level results and the one level results $\left(\phi_{2}-\phi_{1}\right)$ as a function of the one level results for a broad range of input parameters. This figure corresponds to the REAL dataset (Table 2 in the main text defines the range of input values of each dataset). The equivalent plot for the ALL dataset is Figure 3 in the main text. 


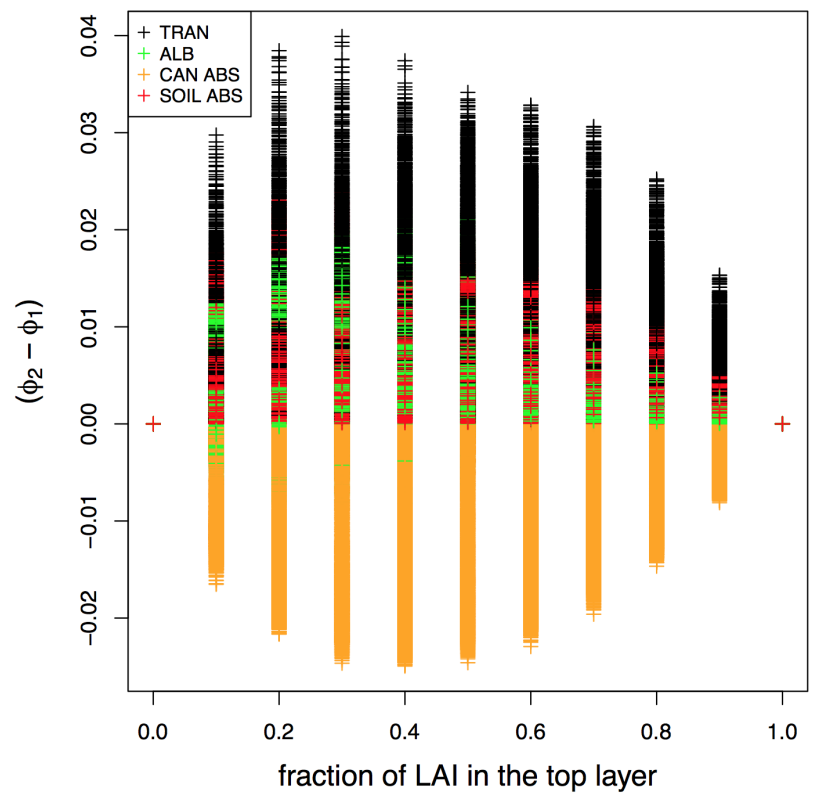

Figure S3: Signed difference for various canopy fluxes of transmission (TRAN), total albedo (ALB), top of the canopy (CAN ALB) and absorption by the soil (SOIL ABS), between the two level results and the single level results $\left(\phi_{2}-\phi_{1}\right)$ as a function of the fraction of LAI in the top level for a broad range of input parameters. This figure corresponds to the REAL dataset (Table 2 in the main text defines the range of input values of each dataset). The equivalent plot for the ALL dataset is Figure 4 in the main text. 

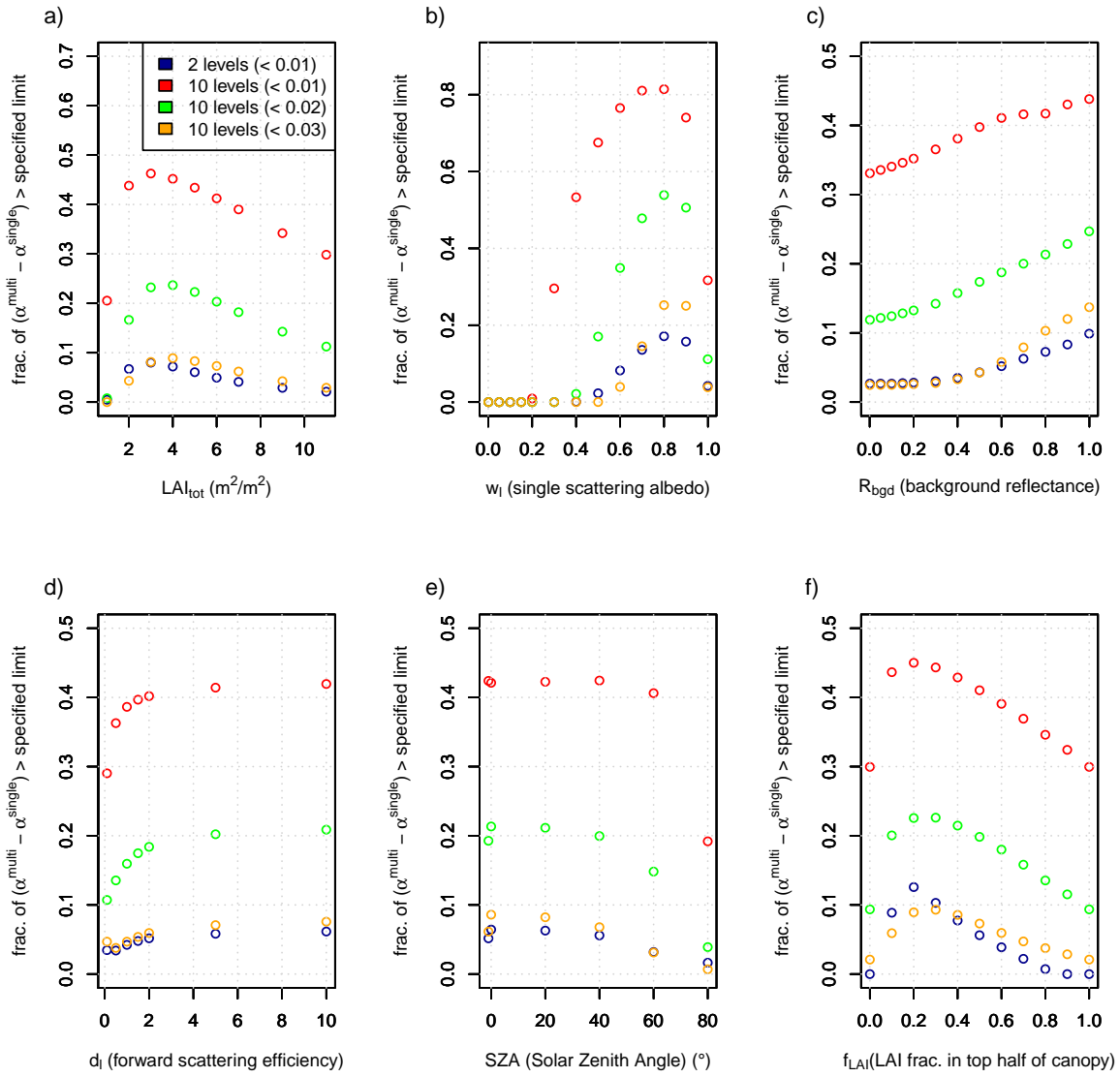

Figure S4: Marginal values of each of the independent input variables against the fraction of points for which the absolute difference of $\left(\alpha_{\text {collim }}^{\text {multi }}-\alpha_{\text {collim }}^{\text {single }}\right)$, is less than 0.01 (red), 0.02 (green) and 0.03 (orange) for the 10 level multi-layer model, and below 0.01 (blue) for the 2 level multilevel model. 

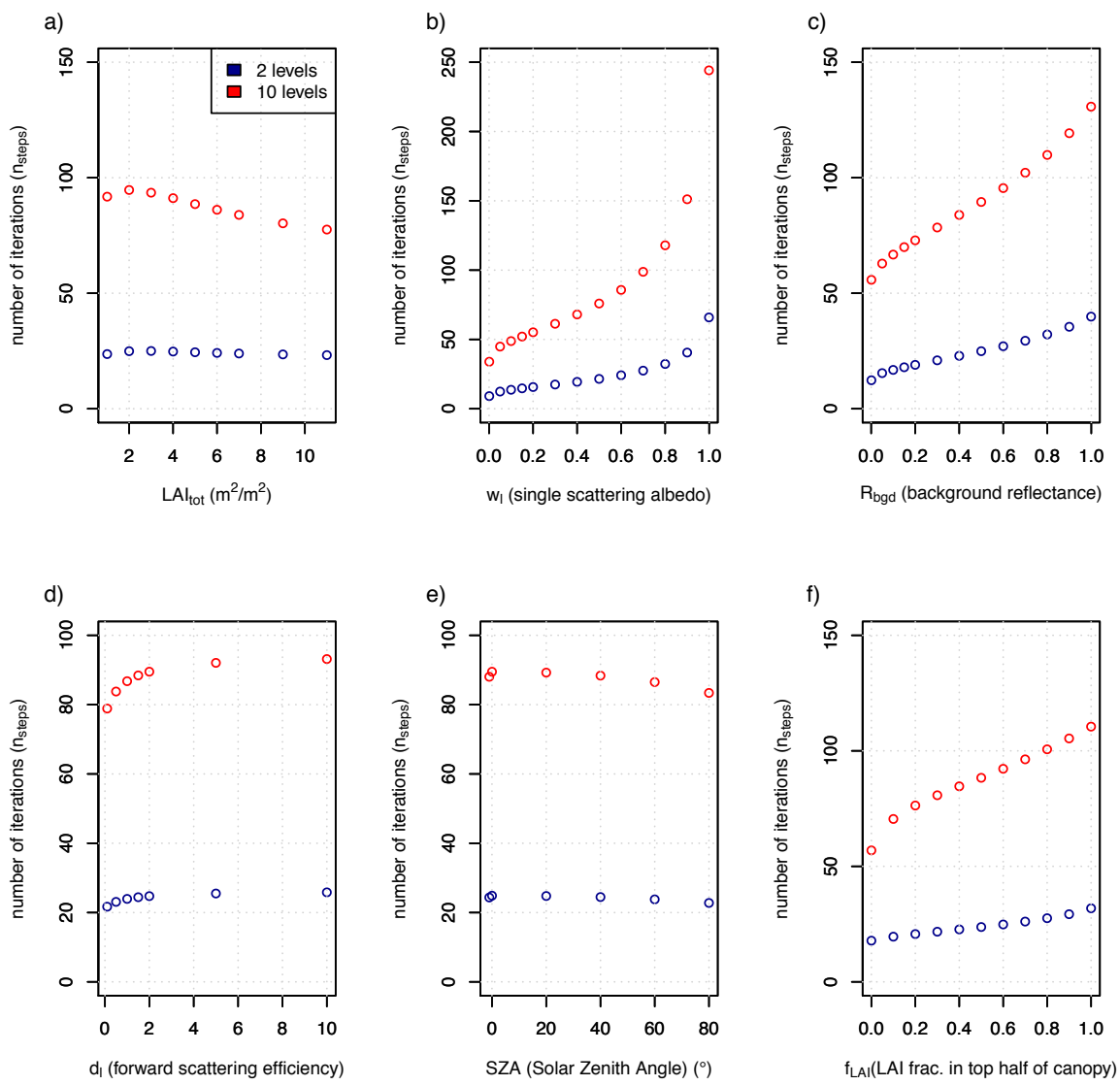

Figure S5: Marginal values for each of the independent input variables against $n_{\text {steps }}$, the total number of iterations required to arrive at a solution, for the ALL dataset, for 2 level model (blue) and the 10 level model (red). 

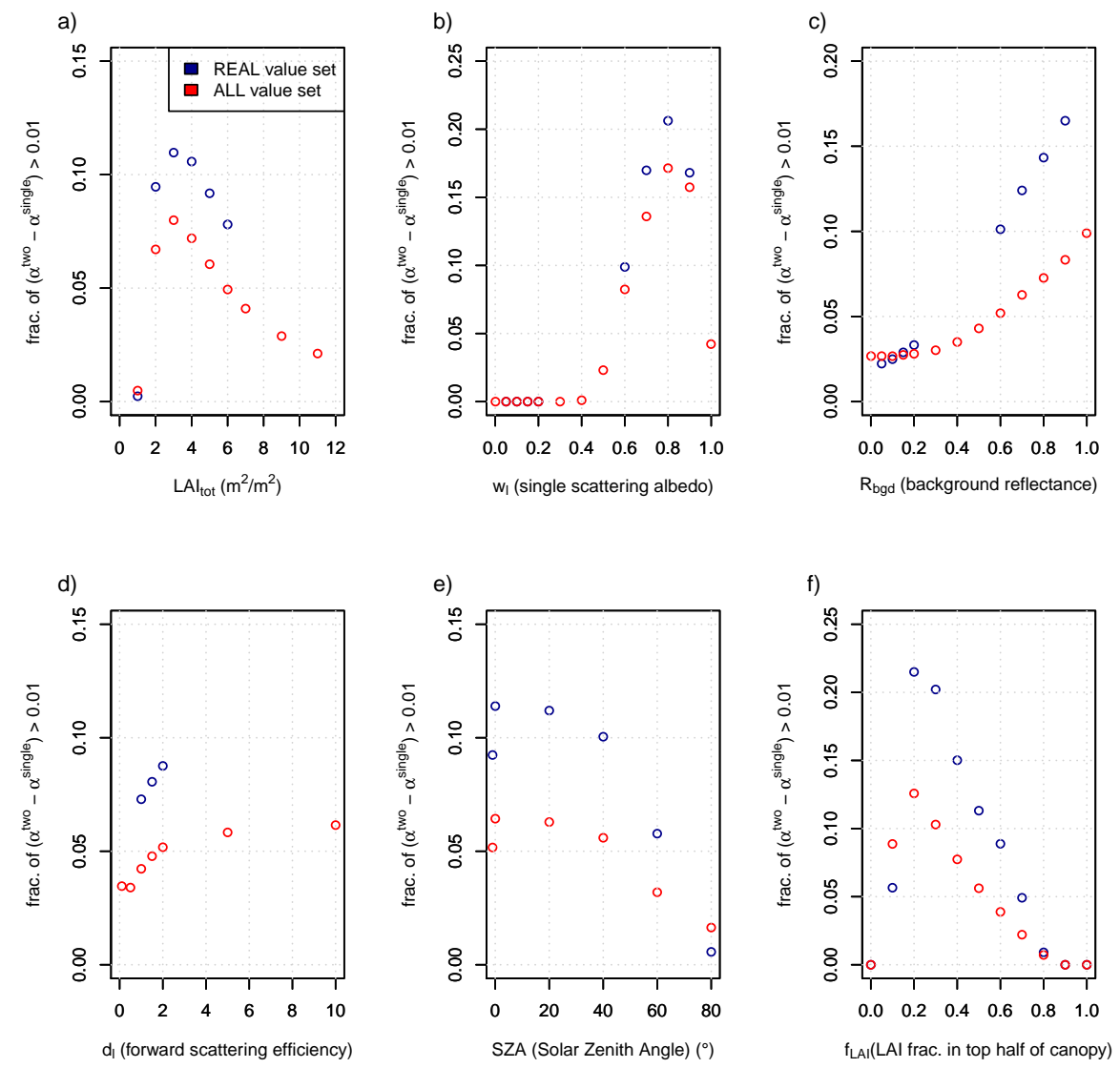

Figure S6: Marginal values of each of the independent input variables against the fraction of points for which the absolute difference of $\left(\alpha_{\text {collim }}^{\text {multi }}-\alpha_{\text {collim }}^{\text {single }}\right)$, is less than 0.01 for the REAL value dataset (blue), and the ALL value dataset (red). 

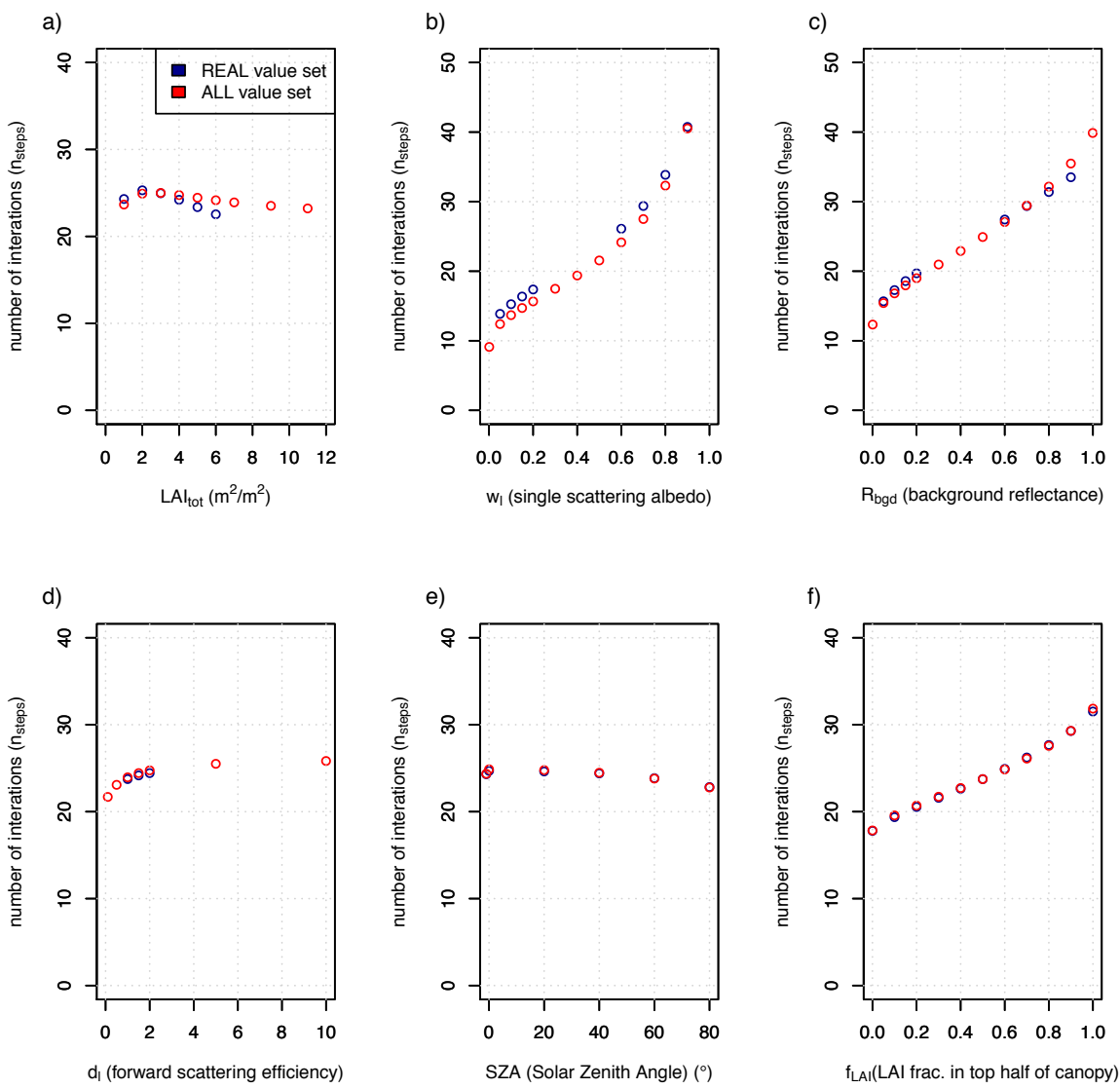

Figure S7: Marginal values for each of the independent input variables against $n_{\text {steps }}$, the total number of iterations required to arrive at a solution for the REAL value dataset (blue), and the ALL value dataset (red). 


\section{List of Tables}

1 Table of the contribution from each input variable to the deviance in the value of $\left(\alpha_{\text {collim }}^{\text {multi }}-\alpha_{\text {collim }}^{\text {single }}\right)$, the difference of the collimated albedo for the multi-level and for the single level model, and of the contribution of each input variable to the deviance in the value of $n_{\text {steps }}$, the total number of iterations required to reach a result in the multi-level model. Same data is displayed in Figures 5 and 6, in the main text . . . . . . . S12 
Table 1: Table of the contribution from each input variable to the deviance in the value of $\left(\alpha_{\text {collim }}^{\text {mult }}-\alpha_{\text {collim }}^{\text {single }}\right)$, the difference of the collimated albedo for the multi-level and for the single level model, and of the contribution of each input variable to the deviance in the value of $n_{\text {steps }}$, the total number of iterations required to reach a result in the multi-level model. Same data is displayed in Figures 5 and 6 , in the main text

\begin{tabular}{lcccc}
\hline \multicolumn{3}{c}{2 levels } & \multicolumn{2}{c}{10 levels } \\
\hline & $\left(\alpha_{\text {collim }}^{\text {mult }} \alpha_{\text {collim }}^{\text {single }}\right)$ & $n_{\text {steps }}$ & $\left(\alpha_{\text {collim }}^{\text {muli }}-\alpha_{\text {collim }}^{\text {single }}\right)$ & $n_{\text {steps }}$ \\
\hline$L A I_{\text {eff }}$ & $3.18 \mathrm{e}-2$ & $2.43 \mathrm{e}-2$ & $7.45 \mathrm{e}-4$ & $5.59 \mathrm{e}-3$ \\
$w_{l}$ & $2.87 \mathrm{e}-1$ & $5.92 \mathrm{e}-1$ & $4.43 \mathrm{e}-1$ & $5.31 \mathrm{e}-1$ \\
$R_{\text {bgd }}$ & $3.07 \mathrm{e}-2$ & $3.72 \mathrm{e}-2$ & $1.31 \mathrm{e}-1$ & $8.55 \mathrm{e}-2$ \\
$d_{l}$ & $6.45 \mathrm{e}-3$ & $1.96 \mathrm{e}-2$ & $3.06 \mathrm{e}-3$ & $3.09 \mathrm{e}-3$ \\
$S Z A$ & $3.33 \mathrm{e}-2$ & $5.44 \mathrm{e}-2$ & $1.05 \mathrm{e}-3$ & $7.62 \mathrm{e}-4$ \\
$f_{L A I, t o t}$ & $1.37 \mathrm{e}-1$ & $1.80 \mathrm{e}-2$ & $3.42 \mathrm{e}-2$ & $4.04 \mathrm{e}-2$ \\
\hline$\left(L A I_{\text {eff }}, w_{l}\right)$ & $3.31 \mathrm{e}-2$ & $4.23 \mathrm{e}-2$ & $6.22 \mathrm{e}-2$ & $7.61 \mathrm{e}-2$ \\
$\left(L A I_{\text {eff }}, R_{b g d}\right)$ & $1.24 \mathrm{e}-2$ & $1.03 \mathrm{e}-2$ & $3.35 \mathrm{e}-3$ & $1.45 \mathrm{e}-3$ \\
$\left(L A I_{\text {eff }}, d_{l}\right)$ & $2.80 \mathrm{e}-4$ & $9.75 \mathrm{e}-4$ & $5.17 \mathrm{e}-6$ & $3.43 \mathrm{e}-6$ \\
$\left(L A I_{\text {eff }}, S Z A\right)$ & $3.05 \mathrm{e}-3$ & $6.26 \mathrm{e}-3$ & $5.89 \mathrm{e}-5$ & $1.18 \mathrm{e}-5$ \\
$\left(L A I_{\text {eff }}, f_{L A I, t o t}\right)$ & $3.94 \mathrm{e}-2$ & $4.97 \mathrm{e}-3$ & $6.95 \mathrm{e}-4$ & $4.02 \mathrm{e}-4$ \\
$\left(w_{l}, R_{b g d}\right)$ & $3.04 \mathrm{e}-2$ & $3.97 \mathrm{e}-2$ & $1.92 \mathrm{e}-1$ & $1.35 \mathrm{e}-1$ \\
$\left(w_{l}, d_{l}\right)$ & $4.24 \mathrm{e}-3$ & $1.11 \mathrm{e}-2$ & $3.16 \mathrm{e}-3$ & $4.69 \mathrm{e}-3$ \\
$\left(w_{l}, S Z A\right)$ & $2.25 \mathrm{e}-2$ & $2.38 \mathrm{e}-5$ & $2.38 \mathrm{e}-5$ & $2.95 \mathrm{e}-5$ \\
$\left(w_{l}, f_{L A I, t o t}\right)$ & $1.22 \mathrm{e}-1$ & $1.10 \mathrm{e}-2$ & $1.88 \mathrm{e}-2$ & $2.33 \mathrm{e}-2$ \\
$\left(R_{b g d}, d_{l}\right)$ & $2.18 \mathrm{e}-3$ & $3.80 \mathrm{e}-3$ & $1.35 \mathrm{e}-3$ & $7.27 \mathrm{e}-4$ \\
$\left(R_{b g d}, S Z A\right)$ & $3.63 \mathrm{e}-5$ & $5.88 \mathrm{e}-5$ & $1.00 \mathrm{e}-4$ & $4.62 \mathrm{e}-5$ \\
$\left(R_{b g d}, f_{L A I, t o t}\right)$ & $1.03 \mathrm{e}-2$ & $1.31 \mathrm{e}-4$ & $1.12 \mathrm{e}-2$ & $1.70 \mathrm{e}-2$ \\
$\left(d_{l}, S Z A\right)$ & $2.27 \mathrm{e}-4$ & $7.75 \mathrm{e}-4$ & $5.06 \mathrm{e}-6$ & $2.32 \mathrm{e}-6$ \\
$\left(d_{l}, f_{L A I, t o t}\right)$ & $2.81 \mathrm{e}-3$ & $6.63 \mathrm{e}-4$ & $1.06 \mathrm{e}-4$ & $1.62 \mathrm{e}-4$ \\
$\left(S Z A, f_{L A I, t o t}\right)$ & $1.07 \mathrm{e}-2$ & $5.01 \mathrm{e}-4$ & $9.36 \mathrm{e}-5$ & $5.98 \mathrm{e}-5$ \\
\hline
\end{tabular}

